\title{
Skeletal muscle deposits from primary oropharyngeal carcinoma: A home away from home
}

\author{
Chinnu Jomi ${ }^{1}$, Preety Negi ${ }^{2}$, Kanwardeep Singh Kwatra ${ }^{3}$, Pamela Alice Kingsley ${ }^{4 *}$ \\ ${ }^{1}$ Junior Resident, ${ }^{2}$ Associate Professor, ${ }^{3,4}$ Professor, ${ }^{\mathbf{1 , 2 , 4}}$ Dept. of Radiation Oncology, ${ }^{3}$ Dept. of Pathology, Christian Medical College \& \\ Hospital, Ludhiana, Punjab, India
}

*Corresponding Author: Pamela Alice Kingsley

Email: pamelajeyaraj@yahoo.co.in

\begin{abstract}
There have been significant improvements in loco-regional control of head and neck squamous cell carcinoma (HNSCC), but distant metastasis remains a clinically important challenge. The incidence of distant metastasis in HNSCC is quite uncommon, varying from $4 \%$ $26 \%$. The most often involved sites being lung, liver and bone. Several factors influencing the incidence of distant metastasis include primary tumour site, grading, nodal involvement, and status of locoregional disease. We report a case of a 59-year old gentleman with oropharyngeal carcinoma treated 6 months back presenting with swelling over the right infra-scapular region and the medial aspect of lower one third of right thigh resulting from metastasis to skeletal muscle. The results of histology and immunohistochemistry were consistent with a metastasis from a squamous cell carcinoma oropharynx. The disease was staged as stage III initially at the time of presentation. The patient received definitive concurrent chemoradiation therapy to the primary and nodal regions. There was no evidence of locoregional disease after 1 year. In the literature, there are reports of HNSCC metastasizing to different parts of the body. However, this is among the few case report of a metastasis to the skeletal muscle.
\end{abstract}

Keywords: Chemoradiotherapy, Immunohistochemistry, Oropharyngeal neoplasm, Skeletal muscle.

\section{Introduction}

More than $90 \%$ of head and neck cancers are squamous cell carcinomas arising from mucosal surfaces of the oral cavity, oropharynx and larynx. Majority of the trials on oropharyngeal squamous cell carcinomas (OPSCC) have shown improvements in the locoregional control due to the better understanding of this cancer in the recent years. ${ }^{1}$ However, unfortunately this improvement in loco-regional control does not seem to have an impact on the survival outcome. $^{2}$ This could be attributed partly to the development of distant metastasis, accounting for approximately $45 \%$ of deaths. ${ }^{3}$ Various factors have been implicated for an increased prevalence of distant metastasis in OPSCC, as the main pattern of therapy failure as compared to local and regional recurrence. These include: achievement of better loco regional control rates thereby, heading the pattern of failure towards distant metastasis. Majority of these patients die of non-oncological causes because patients with OPSCC are younger, healthier with minimal or no tobacco exposure. This results in increase in the proportion of patients who may develop distant metastasis. Further, recent evidence has shown human papilloma virus-associated tumors (HPV) have increased rate of distant metastasis. ${ }^{4}$

Distant metastasis occurs most commonly to the lungs, especially in patients presenting with advanced disease, multiple involved lymph nodes or in the lower neck or in patients with local recurrence. Other metastatic sites being reported include bone, brain, liver, and skin. ${ }^{5}$ The significant variation in the incidence of distant metastasis with $\mathrm{T}$ - and $\mathrm{N}$ stage has been reported; although $\mathrm{N}$ stage has greater influence with no definite relation in the $\mathrm{T}$-size and development of distant metastasis. ${ }^{5}$ To the best of our knowledge, this case report is one of the scantly reported cases of rare sites of oropharyngeal carcinoma metastasis. Herein, we report a case of metastatic oropharyngeal carcinoma to the skeletal muscle of the scapular region and lower extremity.

\section{Case}

A 59-year-old gentleman presented with complaints of painful swelling over the right infra-scapular region and the medial aspect of lower one third of right thigh over a 2 weeks period. The patient was a chronic smoker and alcoholic for the past 40 years.

Detailed history revealed that the patient was diagnosed with HPV-positive squamous cell carcinoma left vallecula (T3N2M0), and he was treated with primary chemoradiation from an outside cancer centre. The patient had received concurrent chemoradiation therapy delivered 5 days a week for 7 weeks at $180 \mathrm{cGy}$ per day with inj. Cisplatin $100 \mathrm{mg} / \mathrm{m}^{2}$ 3-weekly. One year after completing chemoradiotherapy, he presented with painful swelling in the right scapular region and lower one-third of the right thigh. On examination, the patient had an approximately $12 \times 12 \mathrm{~cm}$ well-defined mass in the lower one-third of the right thigh, approximately 3.8 $\mathrm{cm}$ above the medial condyle of tibia, hard in consistency and fixed to underlying muscle (Fig. 1). Another 4 x $3 \mathrm{~cm}$ mass was felt in the right infrascapular region, well-defined, hard in consistency and mobile in all directions located approximately $2 \mathrm{~cm}$ below the angle of scapula (Fig. 2). 


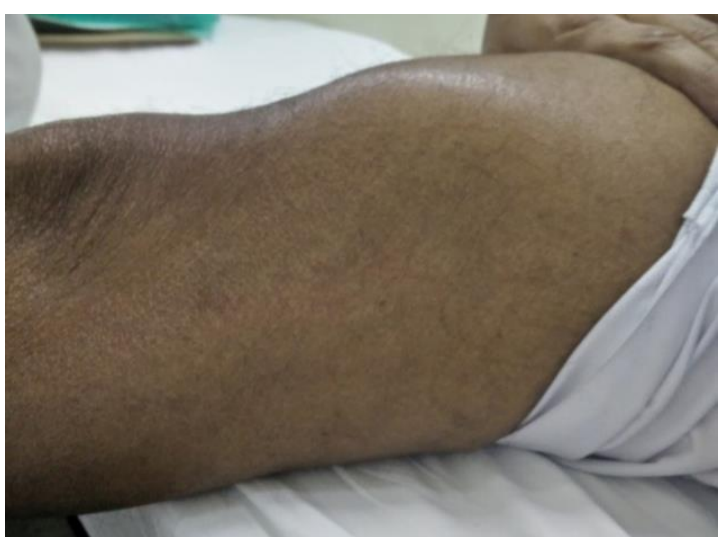

Fig. 1: Clinical picture of thigh swelling on right side

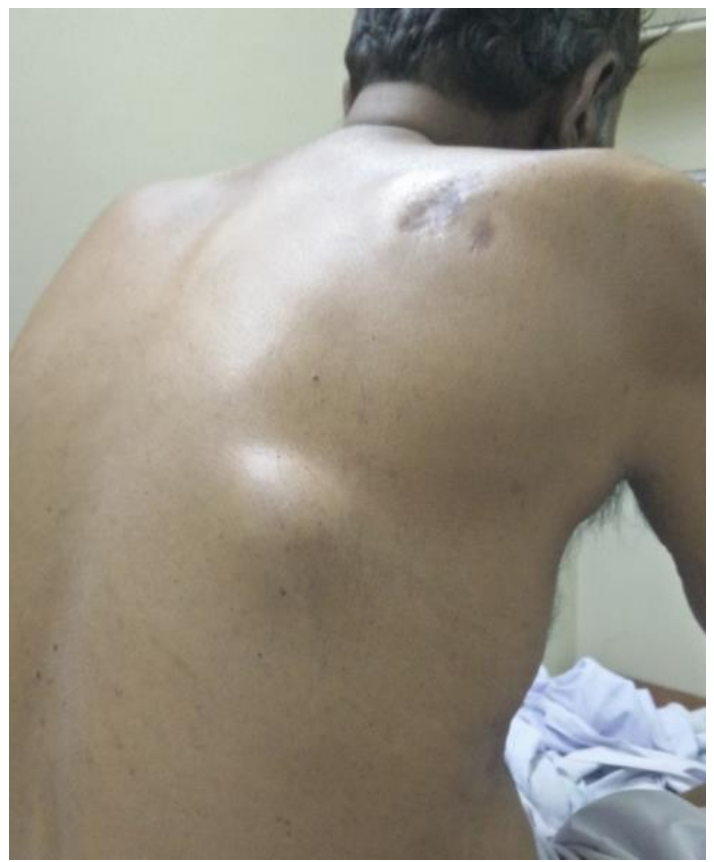

Fig. 2: Clinical picture of swelling in the right infrascapular region

A magnetic resonance imaging (MRI) of right thigh was reported as showing large, well-defined soft tissue lesion in the medial compartment of mid-shaft of right femur, appearing hypointense on $\mathrm{T} 1$ and hyperintense on $\mathrm{T} 2$ weighted / STR images measuring $8.6 \times 4.2 \times 3.9 \mathrm{~cm}$. There was evidence of perilesional edema in the adjoining muscle compartment. The neurovascular bundle was displaced antero-superiorly with no evidence of cortical involvement. Altered marrow signal intensity was seen involving the femoral head and mid-diaphysis (Fig. 5 a-d).
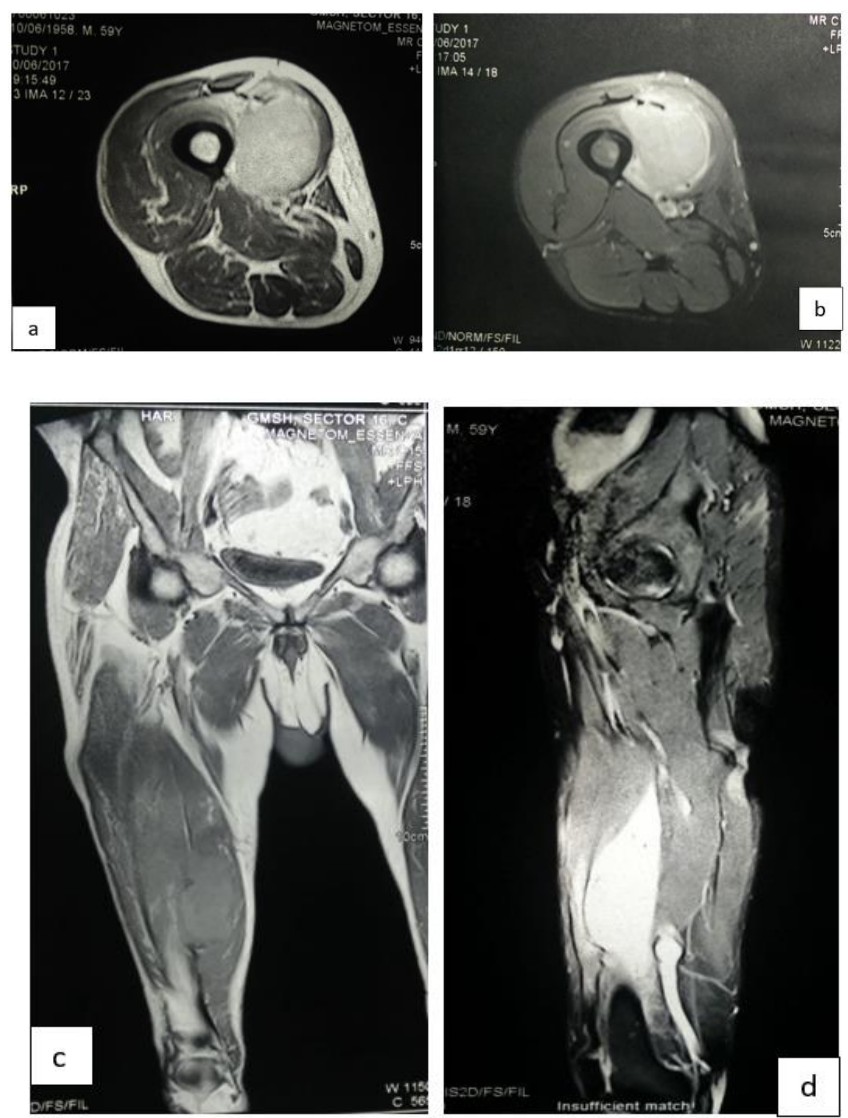

Further imaging by contrast-enhanced computed tomography (CECT) chest and abdomen showed no suspicious lesions in the lung or liver. A workup in the form of MRI face and neck for the primary site of malignancy and evaluation of regional lymph nodes was reported as normal.

Tissue biopsy from the right thigh mass revealed metastatic poorly differentiated adeno-squamous carcinoma. The tumor cells showed immunoreactivity for p63 and CK $5 / 6$ and focal positivity for CK20 (Fig. 3 a-d \& 4). Fine needle aspiration cytology of the right infrascapular mass was consistent with metastatic carcinomatous deposits. The results of histology and immunohistochemistry were reported as consistent with metastasis from a squamous cell carcinoma. The case was discussed in multidisciplinary tumour board and the plan was to begin palliative chemotherapy with paclitaxel and carboplatin. Though the patient did not come for further treatment. 


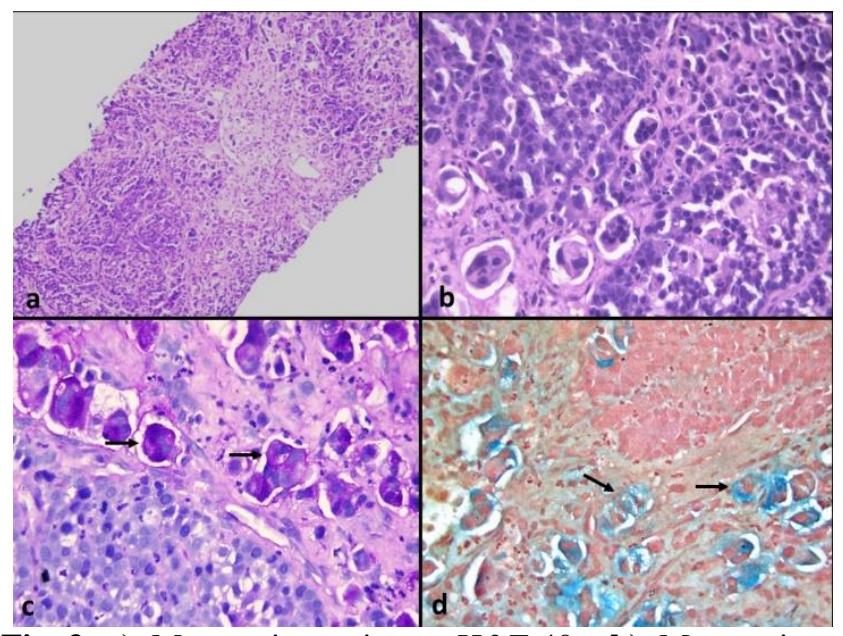

Fig. 3: a): Metastatic carcinoma H\&E 40x; b): Metastatic carcinoma H\&E 400x; c): and d): PAS with diastase, and Alcian blue at $\mathrm{pH} 2.5$ positive for mucin (arrows) respectively in a subset of tumor cells.

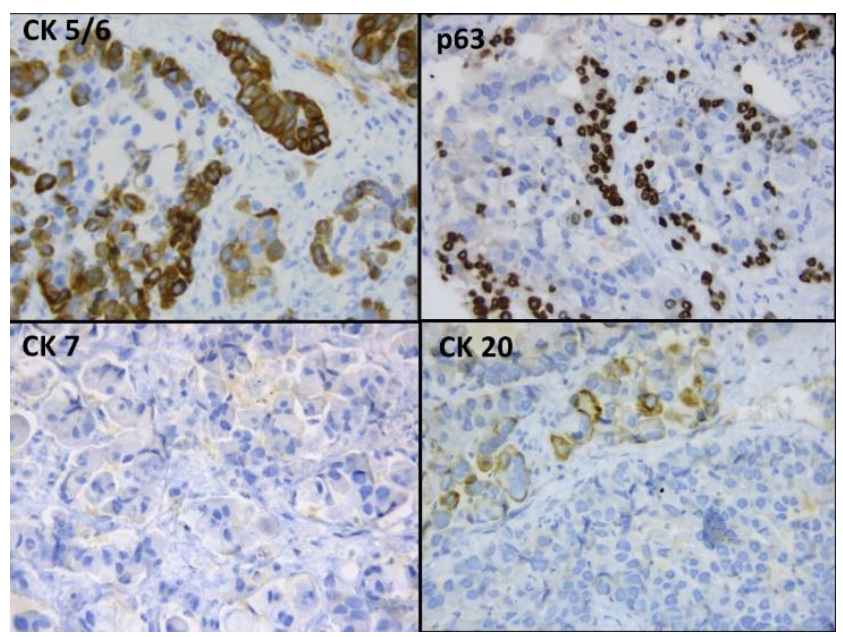

Fig. 4: Immunohistochemistry showing positivity for CK5/6, p63 and focally positivity for CK20. CK7 is negative. DAB chromogen 400x.

\section{Discussion}

The incidence of OPSCC is rapidly increasing worldwide, with an estimated 173,495 new cases in 2018. ${ }^{6}$ Currently, HPV-related OPSCC has been recognized as distinct molecular entity due to its location and role of HPV as etiology. These tumors typically affect younger patients with various collinear sexual activities. Several studies have reported that tumour HPV status is a strong and independent prognostic factor responsible for improved overall survival, progression-free survival, and higher response rates to chemoradiotherapy. ${ }^{7,8}$ Our case had positive history for OPSCC risk factors as smoking, alcohol, and positive HPV status. Felice et al. ${ }^{9}$ have reported that HPV-related OPSCC are more radio-responsive tumors with long-term survival. Keeping this in mind, we can say that our patient did not acquire the benefit of falling under the good prognostic group of HPV-positive OPSCC and thus exhibiting aggressive disease even after radical chemoradiation therapy.
Treatment of locally advanced OPSCC requires a multidisciplinary team approach. Concurrent chemoradiation therapy is the standard of care for definitive management of locally advanced OPSCC since the publication of the several randomized trials and a meta-analysis of chemotherapy in head and neck cancer enrolling more than 17,000 patients. ${ }^{10,11}$ This data showed improvement in overall survival in patients treated with radiotherapy to $70 \mathrm{~Gy}$ along with cisplatin-based chemotherapy as radio-sensitizer (5-year OS; $27.8 \%$ vs $24.3 \%$ without chemotherapy). Our patient had locally advanced OPSCC and was managed with definitive chemoradiation therapy. Inspite of being HPV-negative tumour, this patient had complete response on imaging at 8 weeks after completion of treatment.

Distant metastatic spread from oropharyngeal cancer is relatively uncommon, especially when local control is achieved. ${ }^{12}$ The probability of distant metastasis varies from $15-20 \%$ in head and neck cancer patients. The various risk factors identified for the development of distant metastasis in head and neck carcinoma includes site of the primary tumour, cervical lymph node involvement, grade of tumour, response to treatment, and young age at diagnosis. ${ }^{13}$ Our patient was at higher risk of distant dissemination in view of locally advanced OPSCC stage T3N2M0, moderately differentiated squamous cell carcinoma, rich vascularity of the primary site and HPV-positive status. Literature search revealed that distant metastasis caused by HPV-positive OPSCC tumors often involve unusual anatomical sites resulting in more disseminated disease. ${ }^{14,15}$ These tumors have distant metastasis as the dominant pattern of failure, and may occur after a longer disease-free interval. ${ }^{16}$

The prognosis of squamous cell carcinoma of the head and neck region with distant metastasis is generally very poor. ${ }^{17}$ The common sites of distant metastasis from primary involving oropharyngeal site are lungs followed by bone and liver while metastasis to retroperitoneum, pelvic lymph nodes and other sites is extremely rare. ${ }^{8,19}$ Metastasis to soft tissues involving the lower extremities is reported rarely. ${ }^{20}$ Although it should be kept in mind when dealing with oropharyngeal malignancy presenting with not so common symptoms of metastatic disease since these cells do not follow specified and predetermined path in their journey. We report this case with locally advanced HPV-positive OPSCC with skeletal muscle metastasis, as a rare presentation of metastatic disease from oropharyngeal primary with complete resolution of the primary tumor.

\section{Conclusion}

This case study describes a case of oropharyngeal carcinoma metastasizing to skeletal muscle of the scapular region and lower extremity, an extremely unexpected site of metastasis. We emphasize on the fact that irrespective of the primary tumour site and rarity of occurrence of metastasis, any site could become a fertile soil for the cancer cells to grow and proliferate. In oncology practice, it is of paramount importance to perform a complete and thorough clinical examination during the regular follow-up visits as cancer cells can thrive and home themselves in different 
microenvironments. Clinicians should always raise the possibility of uncommon metastatic presentations in HPVassociated squamous cell carcinoma oropharynx in an attempt to recognize a potential site of metastasis despite a favourable molecular profile.

\section{Source of funding}

None.

\section{Conflict of interest}

None.

\section{References}

1. Pan C, Issaeva N, Yarbrough WG. HPV-driven oropharyngeal cancer: current knowledge of molecular biology and mechanisms of carcinogenesis. Cancers Head Neck 2018;3:12.

2. Garavello W, Ciardo A, Spreafico R, Gaini RM. Risk factors for distant metastasis in head and neck squamous cell carcinoma. Arch Otolaryngol Head Neck Surg 2006;132:7626.

3. Ang KK, Harris J, Wheeler R, Rosenthal DI, Nguyen-Tan PF, Westra WH, et al. human papilloma virus and survival of patients with oropharyngeal cancer. $N$ Engl J Med 2010;363:24-35.

4. Huang SH, Perez-Ordonez B, Weinreb I, Hope A, Massey C, Waldron JN, et al. Natural course of distant metastasis following radiotherapy or chemoradiotherapy in HPV-related oropharyngeal cancer. Oral Oncol 2013;49;79-85.

5. Puthukudy PA, Feshan M. Squamous cell carcinoma of the oropharynx presenting with distant metastasis to ulna. Int $J$ Otolaryngol Head Neck Surg 2013;2:240-4.

6. Bray F, Ferlay J, Soerjomataram I, Siegel RL, Torre LA, Jemal A. Global cancer statistics 2018: GLOBOCAN estimates of incidence and mortality worldwide for 36 cancers in 185 countries. CA: A Cancer J Clin 2018;68:394-424.

7. Swiecicki PL, Malloy KM, Worden FP. Advanced oropharyngeal squamous cell carcinoma: Pathogenesis, treatment, and novel therapeutic approaches. World J Clin Oncol 2016;7:15-26.

8. Ang KK, Harris J, Wheeler R, Weber R, Rosenthal DI, Nguyen-Tan PF, et al. Human papillomavirus and survival of patients with oropharyngeal cancer. $N$ Engl J Med 2010;363:24-35.

9. Felice FD, Tombolini V, Valentini V, Vincentiis M, Mezi S, Brugnoletti $\mathrm{O}$, et al. Advances in the management of HPVrelated oropharyngeal cancer. J Oncol 2019;8.

10. Adelstein DJ, Li Y, Adams GL, Wagner H Jr, Kish JA, Ensley JF, et al. An intergroup phase III comparison of standard radiation therapy and two schedules of concurrent chemoradiotherapy in patients with unresectable squamous cell head and neck cancer. J Clin Oncol 2003;21:92-8.

11. Blanchard P, Baujat B, Holostenco V, Bourredjem A, Baey C, Bourhis J, et al. MACH-NC Collaborative group. Metaanalysis of chemotherapy in head and neck cancer (MACHNC): a comprehensive analysis by tumor site. Radiother Oncol 2011;100:33-40.

12. Kuta V, Rigby M, Hart R, Trites J, Taylor M. Pericardial metastasis from 16 positive oropharyngeal squamous cell carcinoma. Otolaryngol Case Rep 2018;6:25-6.

13. Garavello W, Ciardo A, Spreafico R, Gaini RM. Risk factors for distant metastasis in head and neck squamous cell carcinoma. Arch Otolaryngol Head Neck Surg 2006;132:7626.

14. Daly ME, Le QT, Maxim PG, Loo BW Jr, Kaplan MJ, Fischbein NJ, et al. Intensity-modulated radiotherapy in the treatment of oropharyngeal cancer: clinical outcomes and patterns of failure. Int J Radiat Oncol Biol Phys 2010;76:133946.

15. Huang SH, Perez-Ordonez B, Weinreb I, Hope A, Massey C, Waldron JN, et al. Natural course of distant metastasis following radiotherapy or chemoradiotherapy in HPV-related oropharyngeal cancer. Oral Oncol 2013;49:79-85.

16. Hanna GJ, Kacew A, Chau NG, Shivdasani P, Lorch JH, Uppaluri R, et al. Improved outcomes in PI3K-pathway-altered metastatic HPV oropharyngeal cancer. JCI Insight 2018;3:e122799.

17. Duprez F, Berwouts D, De Neve W, Bonte K, Boterberg T, Deron P, et al. Distant metastasis in head and neck cancer. Head Neck 2017;39:1733-43.

18. Purkayastha A, Sharma N, Suhag V. Extremely rare and unusual case of retroperitoneal and pelvic metastasis from squamous cell carcinoma of vallecula. Int J Cancer Ther Oncol 2016;4:4210.

19. James DZ. Oropharyngeal squamous cell carcinoma metastasis to distal skeletal muscle on FDG PET/CT. Clin Nucl Med 2018;43:e402-3.

20. Samuelian JM, Fisher BJ, Daugherty LC, Babaria UA. Oropharyngeal squamous cell carcinoma metastatic to lowerextremity soft tissues: a case report and literature review. Ear Nose Throat J 2013;92:E38.

How to cite this article: Jomi C, Negi P, Kwatra KS, Kingsley PA. Skeletal muscle deposits from primary oropharyngeal carcinoma: A home away from home. Int $J$ Med Paediatr Oncol 2019;5(4):151-4. 